\title{
BMJ Open Auditory electrophysiological assessments of Alzheimer's disease and preclinical stages: protocol for a systematic review and meta-analysis
}

\author{
Hadeel Y Tarawneh (D) , ${ }^{1,2,3}$ Wilhelmina H A M Mulders, ${ }^{1}$ Hamid R Sohrabi, ${ }^{4,5}$ \\ Ralph N Martins, ${ }^{4,5}$ Dona M P Jayakody ${ }^{2,3}$
}

To cite: Tarawneh HY,

Mulders WHAM,

Sohrabi HR, et al. Auditory electrophysiological assessments of Alzheimer's disease and preclinical stages: protocol for a systematic review and meta-analysis. BMJ Open 2020;10:e033308. doi:10.1136/ bmjopen-2019-033308

- Prepublication history for this paper is available online. To view these files, please visit the journal online (http://dx.doi. org/10.1136/bmjopen-2019033308).

Received 30 July 2019 Revised 03 June 2020 Accepted 10 June 2020

Deck for updates

(c) Author(s) (or their employer(s)) 2020. Re-use permitted under CC BY-NC. No commercial re-use. See rights and permissions. Published by BMJ.

For numbered affiliations see end of article.

Correspondence to Hadeel Y Tarawneh; hadeel.tarawneh@research. uwa.edu.au

\section{ABSTRACT}

Introduction Investigating auditory functions in populations at risk of developing Alzheimer's disease (AD) using auditory neurophysiological measurements can potentially identify a crucial and sensitive diagnostic window of opportunity in preclinical AD. Auditory electrophysiological assessments have gained interest as possible tools for early diagnosis of $A D$. This paper outlines the protocol that will be used to systematically review the published literature currently available on auditory electrophysiological assessments that have been used to assess the auditory functions of adults over the age of 60 years diagnosed with $A D$ or its preclinical stages.

Methods and analysis All full-length peer-reviewed publications of original data that use auditory electrophysiological assessments in $\mathrm{AD}$ and its preclinical stages (subjective cognitive decline (SCD) and mild cognitive impairment (MCI)) will be considered in this review. The search will be performed on major electronic databases (Ovid MEDLINE, Ovid Embase, PsycINF0, PubMed, Scopus and CINAHL Plus) using keywords alone or in combination with Medical Subject Headings divided into two domains; (i) auditory tests and (ii) AD. The database search will be conducted on the $7^{\text {th }}$ of May 2019. Data analysis will be completed and reported in the full review. A random effects meta-analysis will also be conducted using the Comprehensive Meta-Analysis software, V.3. This review will describe which auditory electrophysiological tests have been found to be useful in assessing the auditory function in cognitively impaired adults (MCl and AD) or adults with serious complaints about their cognition (SCD). This review will also identify and describe which auditory electrophysiological test demonstrates the most sensitivity in differentiating people at different stages of cognitive decline.

Ethics and dissemination This systematic review focusses on analysing already available literature. Therefore, there will be no requirement for ethical approval. The systematic review findings will be disseminated through peer-reviewed publication as well as relevant media platforms, for example, conferences.

Systematic review registration PROSPERO CRD42019133553.
Strengths and limitations of this study

- This systematic review protocol follows the Preferred Reporting Items for Systematic Review and MetaAnalysis Protocols (PRISMA-P) guidelines.

- This protocol outlines the procedure for a systematic review which will reduce the possibility of duplication of the methods and allows for peer review of the procedure.

- This will offer highest level evidence for informed decisions.

- The detailed keyword and Medical Subject Headings term search methods offer a more comprehensive search of relevant publications.

- The exclusion of papers that are not published in English may mean some important additional findings are not taken into consideration.

\section{INTRODUCTION}

Worldwide, someone develops dementia every 3 seconds, and in 2017 the number of people estimated to be living with dementia globally was close to 50 million people. ${ }^{1}$ Without a medical breakthrough the number of dementia cases is expected to double every 20 years, with it estimated to be over 152 million cases by $2050 .^{2}$ Contributing to a total of $5.4 \%$ of deaths in males and $10.6 \%$ of deaths in females each year, dementia is the second leading cause of death of Australians. ${ }^{3}$ Everyday approximately 250 Australians are diagnosed with dementia, and this number is expected to increase to 650 people a day by $2056 .^{1}$

Dementia of the Alzheimer's disease (AD) type accounts for $60 \%$ to $80 \%$ of all dementia cases. ${ }^{4}$ It is characterised by loss of episodic memory as well as loss of other cognitive functions. ${ }^{5}$ Cognitive decline has been shown to be strongly associated with hearing loss with the probability of incident dementia log-linearly increasing with the severity of hearing loss. ${ }^{6}$ 
In addition, results from a number of longitudinal studies suggest that changes in central auditory processing skills, even in the absence of severe peripheral hearing loss, are associated with high incidence of cognitive decline and $\mathrm{AD} .{ }^{78}$ Changes in the human auditory system can be objectively measured which can provide an avenue in differentiating normal age-related cognitive dysfunction from $\mathrm{AD}$ and its prodromal states. ${ }^{9-11}$

Investigating peripheral and central auditory functions in populations at risk of developing $\mathrm{AD}$ using auditory neurophysiological measurements combined with audiological assessments can potentially identify a crucial and sensitive diagnostic window of opportunity in preclinical AD. Auditory electrophysiological assessments have gained interest as possible tools for early diagnosis of AD. The present paper outlines the protocol used to systematically review the published literature currently available on auditory electrophysiological assessments that have been used to assess the auditory functions of adults over the age of 65 years diagnosed with $\mathrm{AD}$ and its preclinical stages including those with mild cognitive impairment (MCI) and subjective cognitive decline (SCD). The aims of the systematic review and meta-analysis are; (i) to determine the magnitude of auditory electrophysiological component amplitude and/or latency abnormalities present in $\mathrm{AD}, \mathrm{MCI}$ and SCD when compared with controls, (ii) to determine which auditory electrophysiological component can be used to differentiate between the different study groups (ie, AD, MCI, SCD and controls) and (iii) to determine which auditory electrophysiological assessments can yield a possible diagnostic tool for preclinical $\mathrm{AD}$.

\section{METHODS}

This systematic review will follow the Preferred Reporting Items for Systematic Review and Meta-Analysis (PRISMA) statement. ${ }^{12}$ This protocol is reported in accordance with the PRISMA-Protocols (PRISMA-P) 2015 checklist. ${ }^{13}$ This systematic review is registered in the PROSPERO; International Prospective Register of Systematic Reviews, (http://www.crd.york.ac.uk/PROSPERO/).

\section{Eligibility criteria}

All full-length peer-reviewed publications of original data related to auditory electrophysiological assessments in $\mathrm{AD}$ or its preclinical stages available on the selected databases published between 1985 and May 2019 will be considered in this review.

The eligibility criteria (table 1) illustrate the inclusion and exclusion criteria for publications based on four categories; publication type, population type, assessment type and reported outcome. Only publications of original data that are reported in English will be included in this review, publication of non-original data such as reviews or editorials will not be included in this review. In order to be included in this review all studies must have one or more subject groups with cognitive impairment associated with
$\mathrm{AD}$ and its preclinical stages. The studies must also have used auditory electrophysiological assessments either as a tool for assessing hearing abilities in cognitively impaired adults or as a diagnostic tool for cognitive impairment. Studies using a non-human subject pool and subject groups under the age of 60 will be excluded from this review. The full inclusion and exclusion criteria is illustrated in table 1.

\section{Information sources}

The search will be conducted on the following major databases in medicine, neuroscience and psychology: Ovid Embase, Ovid MEDLINE, PsycINFO, PubMed, Scopus and CINAHL Plus. A hand search of references and citations of the included publications will also be conducted using Google Scholar. In addition, a grey literature search will be conducted using Google Scholar to identify any relevant studies that would not be found through the major database searches.

\section{Search strategy}

Medical Subject Headings (MeSH) in exploded mode in conjunction with keyword searches will be used to maximise the sensitivity of the search strategy in the Embase, MEDLINE and PsycINFO databases. Keyword searches with synonyms, abbreviations, truncations and different spellings (see table 2) will also be used in the PubMed, Scopus and CINAHL Plus databases. The search terms are divided in two domains; (i) auditory tests and (ii) Alzheimer's disease, refer to table 2.

\section{Data management and study selection}

All results from database and hand searches will be exported into EndNote X7 software (Thomson Reuters, 2016), where the duplicate publications will be removed. The study selection will then be undertaken in two stages; first, the titles and abstracts will be screened against the eligibility criteria to confirm whether they are suitable for the final review; second, the full-texts for the eligible publications will be analysed against the eligibility criteria. The search strategy and publication screening will be conducted by two authors (HYT and DMPJ) assisted by Covidence, ${ }^{14}$ a systematic review publication organisational tool (http://www.covidence.org). Any discrepancies between the two authors where a consensus could not be reached will be resolved through discussion and consultation with a third reviewer (WHAMM).

\section{Data items and collection process}

Data extracted from publications for this review will include the following:

1. Publication characteristics: authors, year of publication, journal of publication, title, study objective, study design.

2. Participant characteristics: geographical location of the study, number of participants, age of subject groups, diagnosis method of the disease (SCD or MCI or $\mathrm{AD}$ ), Mini-Mental State Examination score (if reported). 


\begin{tabular}{|c|c|c|}
\hline Eligibility criteria & Include & Exclude \\
\hline \multirow[t]{2}{*}{ Publication type } & - Peer-reviewed, full-length publications of original data. & $\begin{array}{l}\text { Non-original data publications; editorials, } \\
\text { reviews, letters, opinion pieces, } \\
\text { miscellaneous reports, non-empirical } \\
\text { studies. }\end{array}$ \\
\hline & Publications written in English. & $\begin{array}{l}\text { Publications in any language other than } \\
\text { English. }\end{array}$ \\
\hline \multirow[t]{5}{*}{ Population type } & $\begin{array}{l}\text { Studies that include participants with dementia of the } \\
\text { Alzheimer's type and/or its prodromal states. }\end{array}$ & $\begin{array}{l}\text { Studies that only address non-cognitively } \\
\text { impaired adults or adults without memory } \\
\text { complaints. }\end{array}$ \\
\hline & $\begin{array}{l}\text { Studies with participants that are healthy and participants with } \\
\text { cognitive impairment or memory complaints if the data for } \\
\text { cognitively impaired participants is reported. }\end{array}$ & $\begin{array}{l}\text { Studies with no age matched comparison } \\
\text { (control) group. }\end{array}$ \\
\hline & $\begin{array}{l}\text { Studies conducted on humans and other animals if data for } \\
\text { humans is reported. }\end{array}$ & \\
\hline & Studies with an age matched comparison (control) group. & \\
\hline & $\begin{array}{l}\text { Studies on elderly adult participants; mean age of } 60 \text { years } \\
\text { old or older (If mean age not specified the age range for the } \\
\text { subject must start at } 60 \text { years old). }\end{array}$ & \\
\hline Assessment type & $\begin{array}{l}\text { Studies that use auditory electrophysiological assessments } \\
\text { either as a tool for assessing hearing abilities in cognitively } \\
\text { impaired adults or as a diagnostic tool for cognitive } \\
\text { impairment. }\end{array}$ & $\begin{array}{l}\text { Studies that only use auditory assessments } \\
\text { that are not electrophysiological. }\end{array}$ \\
\hline Reported outcome & $\begin{array}{l}\text { Latency and amplitude measures of the auditory } \\
\text { electrophysiological assessments. }\end{array}$ & Self-reported outcomes. \\
\hline
\end{tabular}

AD, Alzheimer's disease; $\mathrm{MCl}$, mild cognitive impairment.

3. Assessment characteristics: testing methods applied, for example, auditory electrophysiology assessment task/paradigm.

4. Outcome characteristics: which auditory electrophysiological measures were used, major findings on components latency and/or amplitude, major conclusions and limitations or difficulties of testing procedures.

The data extracted will be analysed qualitatively to identify any particular patterns, similarities or differences between studies. The data extracted from the publications will provide information about any gaps in knowledge and the future direction of research on auditory electrophysiology in $\mathrm{AD}$ and its preclinical stages.

\section{Quality assessment}

All articles will be assessed to address the risk of; selection bias, performance bias, analysis bias and reporting bias. A quality assessment tool developed by Thomas $e t$ $a l^{15}$ will be used to evaluate the methodological quality of the quantitative studies included in the systematic review. The quality assessment findings will be analysed, and the evidence will be tabulated in order to grade the recommendations based on the American Society of Plastic Surgeons' Evidence Rating Scale for Therapeutic Studies scale for grading recommendations.

\section{Meta-analytic approach}

The meta-analysis will be conducted using the Comprehensive Meta-Analysis (V.3) software developed by Biostat. ${ }^{16}$ The data in each study will be refined for the meta-analysis; (1) grand averages of multiple electrode site measures will be pooled into a single mean for each group, (2) measures reported in subgroups (eg, male and female separately) will be pooled and analysed as a single group, (3) event-related potential (ERP) components reported as subcomponents (eg, P300 as P3a and 


\begin{tabular}{|c|c|c|}
\hline Domain & Keywords & MeSH terms \\
\hline Auditory tests & $\begin{array}{l}\text { psychoacoustic test* OR auditory test OR central auditory test } \\
\text { OR electrophysiological assessment OR electrophysiology OR } \\
\text { electroencephalography OR ECochG OR ECOG OR event related } \\
\text { potential OR ERP OR AEP OR evoked auditory response OR acoustic* } \\
\text { evoked response OR acoustic* evoked potential OR auditory event } \\
\text { related potential OR AERP OR auditory brainstem response OR ABR } \\
\text { OR auditory brainstem responses OR ABRs OR speech-evoked ABR } \\
\text { OR complex ABR OR cABR OR brainstem auditory evoked potential } \\
\text { OR frequency following response OR FFR OR frequency following } \\
\text { potential OR FFP OR envelope following response OR EFR OR auditory } \\
\text { middle latency response OR AMLR OR auditory MLR OR middle latency } \\
\text { response OR middle-latency response OR MLR OR middle latency } \\
\text { responses OR MLRs OR transient middle latency response OR middle } \\
\text { latency auditory evoked potentials OR MLPs OR Na OR Pa OR Nb } \\
\text { OR Pb OR slow vertex potential OR slow-vertex potential OR SVP OR } \\
\text { slow vertex response OR SVR OR V potential OR late cortical response } \\
\text { OR long latency auditory evoked potential OR cortical event-related } \\
\text { potentials OR P1 OR P100 OR N1 OR N100 OR P2 OR P200 OR N2 } \\
\text { OR N200 OR P50 OR late positive component OR late positive complex } \\
\text { OR LPC OR late latency response OR LLR OR slow latency auditory } \\
\text { evoked potentials OR SLAEPs OR late latency auditory evoked potentials } \\
\text { OR LLAEPs OR P300 OR P3 OR mismatched negativity OR MMN OR } \\
\text { mismatched field OR MMF OR contingent negative variation OR CNV } \\
\text { OR N4 OR N400 OR P6 OR P600 OR auditory steady-state response } \\
\text { OR ASSR OR multiple auditory steady-state response OR MASTER OR } \\
\text { multiple-frequency ASSR OR multiple-ASSR OR steady state evoked } \\
\text { response OR SSER OR Steady state evoked potential OR SSEP OR } \\
4 \text { kHz response }\end{array}$ & $\begin{array}{l}\text { Hearing tests OR Auditory evoked } \\
\text { potentials OR Audiometry }\end{array}$ \\
\hline Alzheimer's disease & $\begin{array}{l}\text { Alzheimer* disease OR Alzheimer* OR Dementia OR AD OR cognitive } \\
\text { impairment OR cognitive decline OR cognitive processing OR cognitive } \\
\text { ability dementia OR Alzheimer type dementia OR mild cognitive } \\
\text { impairment OR MCI OR amnestic MCI OR amnestic mild cognitive } \\
\text { impairment OR minimal cognitive impairment OR moderate cognitive } \\
\text { impairment OR severe cognitive impairment OR subjective memory } \\
\text { complainers OR SMC OR subjective cognitive decline OR SCD }\end{array}$ & $\begin{array}{l}\text { Dementia OR Cognitive } \\
\text { dysfunction, impairment OR } \\
\text { Cognition disorders }\end{array}$ \\
\hline
\end{tabular}

P3b) will be combined as a single mean for each group and (4) only baseline measures of the ERP components will be included in the meta-analysis. Standard difference in mean with $95 \%$ CIs will be reported as synthesised measure of effect size. The standard mean difference reflects the difference between the means of two groups divided by their pooled SD. The data for the meta-analysis will be entered as continuous outcomes under the random effects model. The random effects model will be used to account for variation between study methodologies. The q-value statistic will be performed to test heterogeneity of the studies and the $I^{2}$ statistic will indicate heterogeneity as a percentage. A $p$ value $<0.05$ will be considered statistically significant for all analyses.

\section{Synthesis of results}

The data extracted from the studies will be tabulated to illustrate the overall methodological quality, the main findings, the main conclusions and the level of evidence for each study. The findings will be analysed based on the patient group (SCD, MCI or $\mathrm{AD}$ ) and the clinical testing approach (type of auditory electrophysiological assessment and type of outcome measures) in order to synthesise the evidence. An understanding of the feasibility, efficiency, effectiveness and quality of the use of each auditory electrophysiological assessment in the patient groups will be developed through the synthesis of the results.

\section{Patient and public involvement}

Patient and public involvement is not required for this systematic review.

\section{ETHICS AND DISSEMINATION}

The aim of this review is to investigate and describe the different auditory electrophysiological tests that have been used in clinical studies in participants with SCD, $\mathrm{MCI}$ and $\mathrm{AD}$. This review will provide information about which auditory electrophysiological tests have been found to be useful in assessing auditory function in cognitively impaired adults. Additionally, the review will identify the strengths and limitations of each auditory electrophysiological test and identify which auditory electrophysiological test demonstrates most sensitivity in differentiating subject groups; that is, between those at different stages 
of cognitive impairment. This review will also contribute to the study design of clinical studies looking at auditory evoked potentials in patients with $\mathrm{AD}$ and its preclinical stages. The full review and meta-analysis will be submitted for publication in a peer-reviewed journal and the results will be presented at conferences and meetings relevant to the field. Ethical approval is not required as no primary data will be collected.

\section{Author affiliations}

${ }^{1}$ School of Human Sciences, University of Western Australia, Crawley, Western Australia, Australia

${ }^{2}$ Ear Science Institute Australia, Subiaco, Western Australia, Australia

${ }^{3}$ Ear Science Centre, School of Surgery, University of Western Australia, Crawley, Western Australia, Australia

${ }^{4}$ School of Medical and Health Sciences, Edith Cowan University, Joondalup, Western Australia, Australia

${ }^{5}$ Department of Biomedical Sciences, Faculty of Medicine and Health Sciences, Macquarie University, Macquarie, New South Wales, Australia

Contributors HYT, DMPJ, WHAMM, HRS and RNM conceived the idea for this systematic review. HYT prepared the initial protocol draft with input from DMPJ, WHAMM and HRS. All authors contributed to the development of the idea of this systematic review, as well as contributed to the revision of the manuscript. All authors approved for the manuscript to be submitted.

Funding The authors have not declared a specific grant for this research from any funding agency in the public, commercial or not-for-profit sectors.

Patient and public involvement Patients and/or the public were not involved in the design, or conduct, or reporting, or dissemination plans of this research.

Patient consent for publication Not required.

Provenance and peer review Not commissioned; externally peer reviewed.

Open access This is an open access article distributed in accordance with the Creative Commons Attribution Non Commercial (CC BY-NC 4.0) license, which permits others to distribute, remix, adapt, build upon this work non-commercially, and license their derivative works on different terms, provided the original work is properly cited, appropriate credit is given, any changes made indicated, and the use is non-commercial. See: http://creativecommons.org/licenses/by-nc/4.0/.

\section{ORCID iD}

Hadeel Y Tarawneh http://orcid.org/0000-0003-1860-9167

\section{REFERENCES}

1 Brown L, Hansnata E, La H. Economic cost of dementia in Australia 2016-2056, 2017. Available: https://www.dementia.org.au/files/ NATIONAL/documents/The-economic-cost-of-dementia-in-Australia2016-to-2056.pdf

2 Alzheimer's Disease International. World Alzheimer report 2018 the state of the art of dementia research: new frontiers, 2018. Available: https://www.alz.co.uk/research/WorldAlzheimerReport2018.pdf

3 Australian Bureau of Statistics. Australian Bureau of statistics: 3303.0 causes of death, Australia, 2016. Canberra Australian Bureau of Statistics, 2017. Available: https://www.abs.gov.au/ausstats/abs@. nsf/Lookup/by\%20Subject/3303.0 2017 Main\%20Features Australia's\%20leading\%20causes\%20of\%20death,\%202017 2

4 Alzheimer Association. 2017 Alzheimer's disease facts and figures. Alzheimer Dement 2017;13:325-73.

5 McKhann GM, Knopman DS, Chertkow $\mathrm{H}$, et al. The diagnosis of dementia due to Alzheimer's disease: recommendations from the National Institute on Aging-Alzheimer's association workgroups on diagnostic guidelines for Alzheimer's disease. Alzheimers Dement 2011;7:263-9.

6 Lin FR, Metter EJ, O'Brien RJ, et al. Hearing loss and incident dementia. Arch Neurol 2011;68:214-20.

7 Gates GA, Anderson ML, Feeney MP, et al. Central auditory dysfunction in older persons with memory impairment or Alzheimer dementia. Arch Otolaryngol Head Neck Surg 2008;134:771-7.

8 Gates GA, Anderson ML, McCurry SM, et al. Central auditory dysfunction as a harbinger of Alzheimer dementia. Arch Otolaryngol Head Neck Surg 2011;137:390-5.

9 Hardy CJD, Marshall CR, Golden HL, et al. Hearing and dementia. $J$ Neurol 2016;263:2339-54.

10 Taljaard DS, Olaithe M, Brennan-Jones CG, et al. The relationship between hearing impairment and cognitive function: a meta-analysis in adults. Clin Otolaryngol 2016;41:718-29.

11 Vecchio F, Määttä S. The use of auditory event-related potentials in Alzheimer's disease diagnosis. Int $\mathrm{J}$ Alzheimers Dis 2011;2011:653173.

12 Moher D, Liberati A, Tetzlaff J, et al. Preferred reporting items for systematic reviews and meta-analyses: the PRISMA statement. PLoS Med 2009;6:e1000097.

13 Moher D, Shamseer L, Clarke M, et al. Preferred reporting items for systematic review and meta-analysis protocols (PRISMA-P) 2015 statement. Syst Rev 2015;4:1.

14 Covidence. Covidence systematic review software [online], 2019. Available: www.covidence.org

15 Thomas BH, Ciliska D, Dobbins M, et al. A process for systematically reviewing the literature: providing the research evidence for public health nursing interventions. Worldviews Evid Based Nurs 2004;1:176-84.

16 Borenstein M, Hedges LV, Higgins JPT, et al. Comprehensive metaanalysis. 3rd ed. Englewood: Biostat, 2006. 